\title{
Optimum Simultaneous DG and Capacitor Placement on the Basis of Minimization of Power Losses
}

\author{
M. M. Aman, G. B. Jasmon, K. H. Solangi, A. H. A. Bakar, and H. Mokhlis
}

\begin{abstract}
Optimum Distributed Generation (DG) placement and sizing is one of the current topics in new restructured power system. Most of the authors have worked out either DG unit placement or shunt capacitor placement as criteria for minimization of power system losses. However in the present paper, both approaches have been combined together to achieve overall minimum power losses and better voltage regulation. Particle Swarm Optimization (PSO) algorithm will be used for simultaneous finding of optimum DG and shunt capacitor bank location and size. The proposed algorithm will be tested on 12-bus, 30-bus, 33-bus and 69-bus radial distribution networks and the results will also be discussed in detail.
\end{abstract}

Index Terms-DG unit placement, shunt capacitor, particle swarm optimization (PSO), distribution system, power losses.

\section{INTRODUCTION}

With the advent of restructuring in power system, many power companies are investing in small scale distributed power generation. Distributed Generation (DG) have many different types ranging from conventional fossil fuel based combustion engines to the renewable energy including wind, photo-voltaic cells, micro-turbines, small hydro turbines, CHP or hybrid. Different authors have given different definitions of DG, in different perspective. For the purpose of this paper, the "distributed generation is an electric power source connected directly to the distribution network or on the consumer side of the meter" [1].

The word distributed generation is also subjective and changes with respect to the region. For example, 'embedded generation' term is used in Anglo-American countries, 'dispersed generation' is used in North American countries, and 'decentralized generation' term is used in Europe and parts of Asia [1].

Distributed Generation (DG) has many advantages over centralized power generation. The optimum DG placement and sizing at planning stage of distribution system is necessary to achieve the maximum benefits. The non-optimum DG placement and sizinag could results in increase power losses, thus making the voltage profile lower than the allowable voltage limit [2].

Manuscript received February 23, 2013; revised April 27, 2013. This work was supported by Bright Spark Program of University of Malaya and NED University of Engineering and Technology.

M. M. Aman is with NED University of Engineering and Technology, Karachi, Pakistan. He is also doing Ph.D. from University of Malaya (e-mail: mmohsin@neduet.edu.pk).

G. B. Jasmon and K. H. Solangi are with University of Malaya, Kuala Lumpur, Malaysia.

A. H. A. Bakar and H. Mokhlis are working under the Faculty of Engineering, University of Malaya, Kuala Lumpur, Malaysia.
The increment in active power loss represents loss in savings to the utility as well as a reduction in feeder utilization. Studies have shown that $70 \%$ of power losses are due to distribution system [3] and the losses resulting from Joule effect only account for $13 \%$ of the generated energy [4]. This non-negligible amount of losses has a direct impact on the financial results and the overall efficiency of the system. The unbundling of utility has forced the distribution companies to reduce the losses and operate at the highest efficiency for their own economic benefits. Since utilities are already facing technical and non-technical issues, they cannot tolerate such additional issues. Hence an optimum placement of DG is needed in order to minimize overall system losses and therefore improve voltage profiles [5]- [7].

DG is mainly considered as an active source of energy $(\mathrm{kWh})$ to get the maximum benefit. Companies are investing in distribution system to meet the active power demand to get the maximum profit. However, drawing maximum active power can results into deficiency in fulfilling the kVAr of reactive load requirement. This situation can become worst and voltage collapse condition can occur. Thus it is necessary to optimally place the shunt capacitors for reactive power support in the distribution system. Optimal capacitor placement and sizing also helps in reduction of losses and increases power transfer capability on congested transmission system. The recent power blackouts, due to insufficient reactive power, have also increased the importance of meeting reactive power demand of the system locally. The stability of voltage can be maintained by providing reactive power support to the distribution system at optimum locations locally [8], [9].

In this paper, the above problem is solved by simultaneous DG and capacitor bank placement in distribution system, considering minimization of power losses as fitness function. Voltage limits $\left(V_{\min }\right.$ and $\left.V_{\max }\right)$ is considered as one of the system constraints.

This paper is organized as follows: In section 2, problem formulation will be presented. Section 3, will briefly discuss about the Particle Swarm Optimization (PSO) algorithm. In section 4, the proposed algorithm will be tested on four different radial distribution test systems.

\section{PROBlem Formulation}

In the proposed algorithm of simultaneous DG and capacitor placement, the fitness function considered is minimization of total active power losses $\left(P_{L}\right)$, given by Eqn. (1).

$$
f=\operatorname{Min}\left\{P_{L}=\sum_{i=1}^{n}\left|I_{i}\right|^{2} R_{i}\right\}
$$


where,

$I$ is the line current in branch $i$.

$R$ is the resistance of branch $i$.

$\mathrm{N}$ is total number of branches.

Subjected to the following generation constraints:

$$
\begin{gathered}
0 \leq P_{d g} \leq \sum P_{\text {load }} \\
0 \leq Q_{\text {shunt }} \leq \sum Q_{\text {slack }}
\end{gathered}
$$

Other system constraints include bus voltages $\left(V_{\text {bus }}\right)$ :

$$
1.05 \leq V_{b u s}^{n} \leq 0.95
$$

where

$V_{\text {bus }}$ is $n^{\text {th }}$ bus voltage.

To find the optimum DG and capacitor placement and corresponding size, Particle Swarm Optimization (PSO) algorithm is used here. The brief introduction of PSO algorithm is presented in section 3 .

\section{Particle Swarm Optimization (PSO) Algorithm}

Particle Swarm Optimization (PSO) is introduced by [10] to solve the optimization problem, based on social-psychological 'bird flock's behaviour. In this case, social sharing of information takes place and individuals can take profit from the findings and experience of all the other companions during the search for food. Each companion, called particle, in the population, which is called swarm, is assumed to 'fly' over the search space ' $\mathrm{S}$ ' in order to find promising regions of the landscape.

The complete illustration of PSO algorithm is shown in Fig. 2. A particle $i$ is represented as $x_{i}=\left(x_{i 1}, x_{i 2}, x_{i 3}, x_{i 4}, \ldots \ldots\right.$, $\left.x_{i \mathrm{~d}}\right)$. The position associated with the best fitness pbesti $=\left(\right.$ pbest $_{\mathrm{i} 1}$, pbest ${ }_{\mathrm{i} 2}$, pbest ${ }_{\mathrm{i} 3}$, pbest ${ }_{\mathrm{i} 4} \ldots \ldots \ldots$..... pbest $\left.{ }_{\mathrm{id}}\right)$ is considered as its current best position. Here $d$ is a total number of initial particles. The overall best particle among all particles in the group is recorded as $g_{\text {best }}$.

The new particle position is based on the rate of the position of $i^{\text {th }}$ particle, represented as $v_{i}=\left(v_{i 1}, v_{i 2}, v_{i 3}, v_{i 4}\right.$, $\left.\ldots \ldots . v i_{d}\right)$. During the iteration procedure, the velocity and the position of $i^{\text {th }}$ particles are updated according to the Eqns. (6-7):

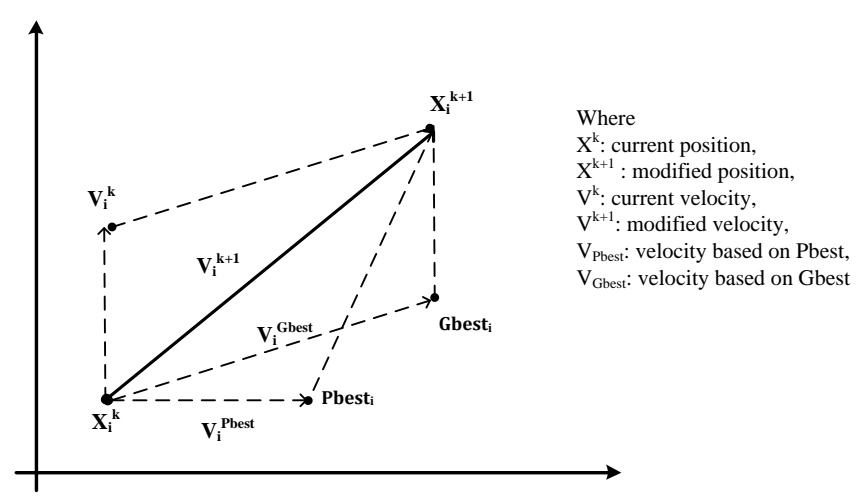

Fig. 1. Magnetization as a function of applied field.

$$
\begin{gathered}
v_{i d}{ }^{(t+1)}=w \cdot v_{i d}{ }^{(t)}+c_{1} \cdot r_{1} \cdot\left(\text { pbest }_{i d}-x_{i d}\right)+c_{2} \cdot r_{2} \cdot\left(\text { gbest }-x_{i d}\right) \\
x_{i d}{ }^{(t+1)}=x_{i d}{ }^{(t)}+v_{i d}{ }^{(t+1)}
\end{gathered}
$$

where,

$t$ is number of iterations, $c_{1}$ and $c_{2}$ are acceleration constants (usually taken as 0.7), $r_{1}$ and $r_{2}$ are random numbers, $w$ is inertia weight given by Eqn. (8).

$$
w=w_{\max }+\frac{w_{\max }-w_{\min }}{\text { iter }} \times t
$$

where,

$w_{\max }$ and $w_{\min }$ is 0.9 and 0.4 respectively, $\mathrm{c}_{1}$ and $\mathrm{c}_{2}$ are constants (0.7).

In the present case of DG placement and sizing, the $i^{\text {th }}$ particle $\left(x_{i}\right)$ is a four dimension vector $\left(\mathrm{P}_{\mathrm{DG}}, \mathrm{P}_{\mathrm{DG}}, \mathrm{Q}_{\text {Shunt }}\right.$, $\mathrm{Q}_{\text {Shunt }}$ ), representing random $\mathrm{DG}$ and shunt capacitor positions $\left(\mathrm{P}_{\mathrm{DG}}, \mathrm{Q}_{\text {Shunt }}\right)$ and $\mathrm{DG}$ and shunt capacitor sizes $\left(\mathrm{P}_{\mathrm{DG}}\right.$, QShunt).

\section{PROPOSED AlgorithM}

Table I shows the complete steps of the proposed

\begin{tabular}{|c|c|}
\hline Steps & Discussion \\
\hline Initialization & $\begin{array}{l}\text { - Initialize PSO parameters } \\
\text { - A particle } i \text { among initial populations }(\mathrm{d}) \text { is a six } \\
\text { dimension position vector } \mathrm{x}_{\mathrm{i}} \text { representing DG } \\
\text { positions }\left(\mathrm{P}_{\mathrm{DG}}\right) \text {, Shunt Capacitor positions } \\
\left(\mathrm{Q}_{\text {Shunt }}\right), \mathrm{DG} \text { sizes }\left(\mathbf{P}_{\mathrm{DG}}\right) \text { and Shunt Capacitor size } \\
\left(\mathbf{Q}_{\text {Shunt }}\right) \\
\mathrm{X}_{\mathrm{i}}=\left[\mathrm{P}_{\mathrm{DG}}, \mathbf{P}_{\mathrm{DG}}, \mathrm{Q}_{\text {Shunt }}, \mathbf{Q}_{\text {Shunt }}\right]\end{array}$ \\
\hline $\begin{array}{c}\text { Generate Initial } \\
\text { Solutions }\end{array}$ & $\begin{array}{l}\text { Calculate the best fitness function (Eqn. (1)) among } \\
\text { initial solution of } x_{i} \text {, considering the constraints given } \\
\text { in Eqns. (2)-(4). }\end{array}$ \\
\hline $\begin{array}{l}\text { Update Initial } \\
\mathbf{p}_{\text {best }} \text { and } \mathbf{g}_{\text {best }}\end{array}$ & For Iteration $=1$, find initial $\mathrm{p}_{\text {best }}$ and $\mathrm{g}_{\text {best }}$. \\
\hline $\begin{array}{l}\text { Generate new } \\
\text { set of positions }\end{array}$ & $\begin{array}{l}\text { - Update velocity and position vectors using Eqns. } \\
(5-6) \\
\text { - Check the feasibility of the new generated } \\
\text { particles xi, given in Eqns. (2)-(4). }\end{array}$ \\
\hline $\begin{array}{c}\text { Update Initial } \\
\mathbf{p}_{\text {best }} \text { and } g_{\text {best }}\end{array}$ & $\begin{array}{l}\text { - Calculate new fitness function of updated } \\
\text { particles. } \\
\text { - Update } p_{\text {best }} \text { and } g_{\text {best. }}\end{array}$ \\
\hline Stopping Criteria & $\begin{array}{l}\text { Update Iteration } \\
\text { If } \\
\text { Iteration }==\text { Iteration }_{(\max )} \\
\text { End }\end{array}$ \\
\hline
\end{tabular}
algorithm for optimum DG and shunt capacitor placement and sizing. Thukaram load flow is used to carry out the load flow analysis.

TABLE I: PROPOSED ALGORITHM FOR SIMULTANEOUS OPTIMUM DG AND CAPACITOR PLACEMENT

\section{RESUlTS AND DISCUSSION}

The proposed algorithm is tested on 12-bus [11], 30-bus [12], 33-bus [13] and 69-bus [14] radial distribution systems.

\section{A. Results}

Using the proposed method following optimum DG and shunt capacitor locations and their corresponding sizes have been calculated. The results are tabulated in Table II. 
TABLE II: APPLICATION OF PROPOSED ALGORITHM FOR SiMULTANEOUS DG AND CAPACITOR PLACEMENT AND SIZING

\begin{tabular}{ccccc}
\hline \hline \multirow{2}{*}{$\begin{array}{c}\text { Test } \\
\text { System }\end{array}$} & $\begin{array}{c}\text { DG } \\
\text { Position }\end{array}$ & $\begin{array}{c}\text { DG } \\
\text { Size(MW) }\end{array}$ & $\begin{array}{c}\text { Shunt } \\
\text { Capacitor } \\
\text { Position }\end{array}$ & $\begin{array}{c}\text { Shunt } \\
\text { Capacitor } \\
\text { Size(MW) }\end{array}$ \\
\hline 12-Bus & 9 & 0.2339 & 9 & 0.2107 \\
30-Bus & 21 & 5.7553 & 21 & 3.4378 \\
33-Bus & 6 & 2.5106 & 30 & 1.4571 \\
69-Bus & 61 & 1.5660 & 61 & 1.4013 \\
\hline \hline
\end{tabular}

\section{B. Discussion}

The effect of DG and shunt capacitor placement is also measured by calculating the power system losses and system loading factor. Table III shows the effect of DG placement on system performance.

TABLE III: Effect of Proposed Algorithm for Simultaneous DG and

\begin{tabular}{ccccc}
\hline & \multicolumn{2}{c}{ Capacitor Placement and Sizing } \\
\cline { 2 - 5 } Test & \multicolumn{2}{c}{ Without DG } & \multicolumn{2}{c}{ Proposed Method } \\
\cline { 2 - 5 } System & $\begin{array}{c}\text { System } \\
\text { Loadability }\end{array}$ & $\begin{array}{c}\text { System } \\
\text { Losses } \\
(\mathrm{MW})\end{array}$ & $\begin{array}{c}\text { System } \\
\text { Loadability }\end{array}$ & $\begin{array}{c}\text { System } \\
\text { Losses } \\
(\mathrm{MW})\end{array}$ \\
\hline 12-Bus & 5.33 & 0.0207 & 6.33 & 0.0032 \\
30-Bus & 2.78 & 0.8819 & 3.58 & 0.1754 \\
33-Bus & 3.40 & 0.2110 & 3.93 & 0.0597 \\
69-Bus & 3.20 & 0.2250 & 4.18 & 0.0259 \\
\hline \hline
\end{tabular}

From Table III, it can be seen that the system losses have been considerably reduced and system loading capability has significantly improved. These results are also shown in Fig. 2 and Fig. 3.

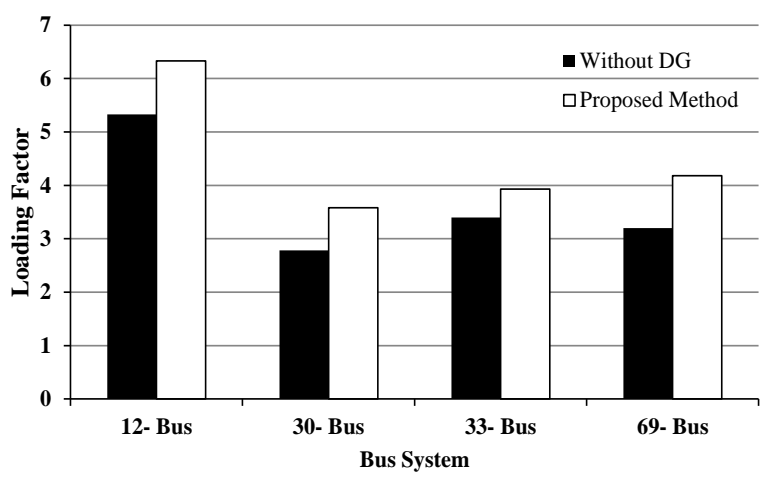

Fig. 2. System Loading Improvement due to DG Placement

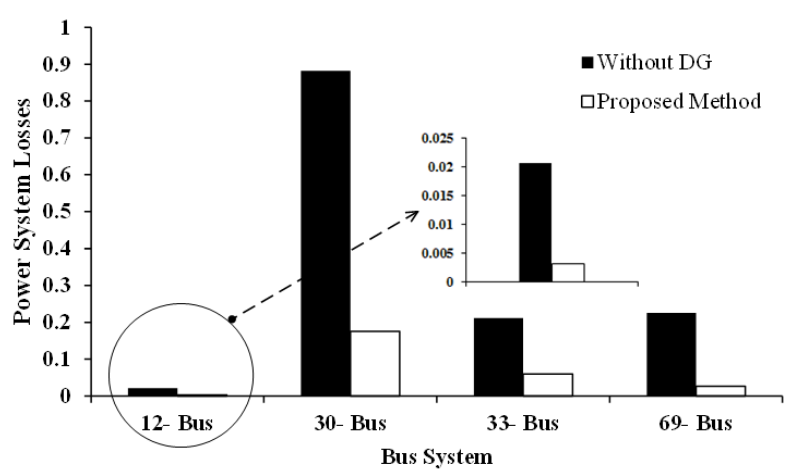

Fig. 3. System Loading Improvement due to DG and Capacitor Placement

Fig. 4(a-d) shows the voltage profile improvement for all test systems.

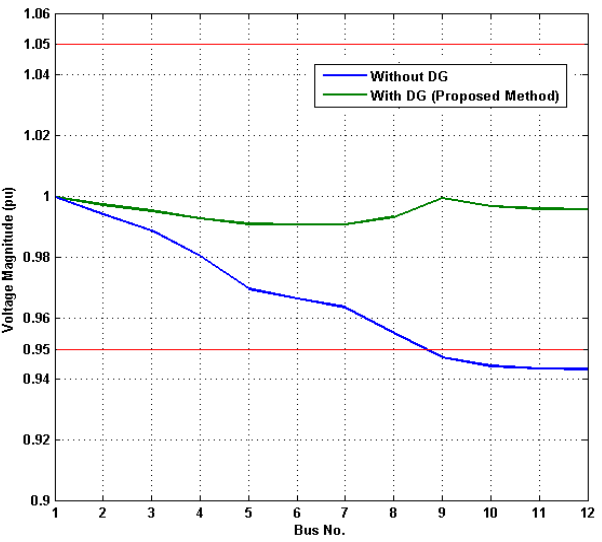

(a) 12-Bus System

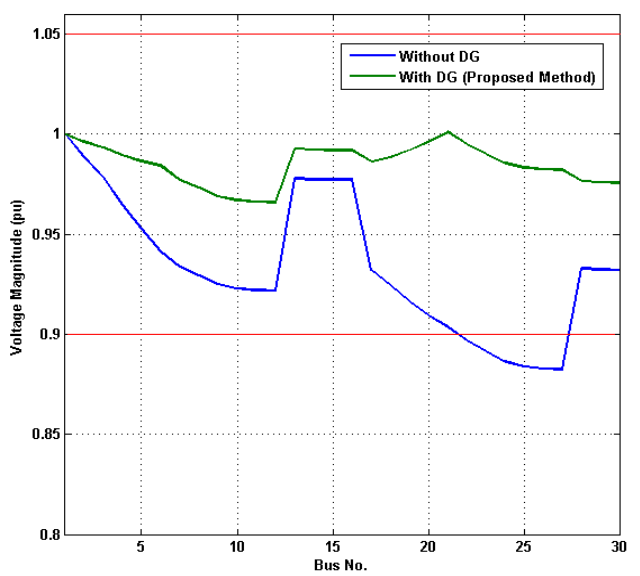

(b) 30-Bus System

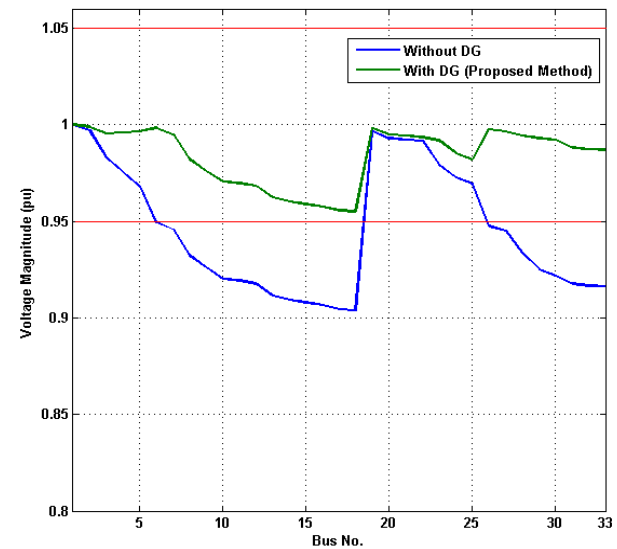

(c) 33-Bus System

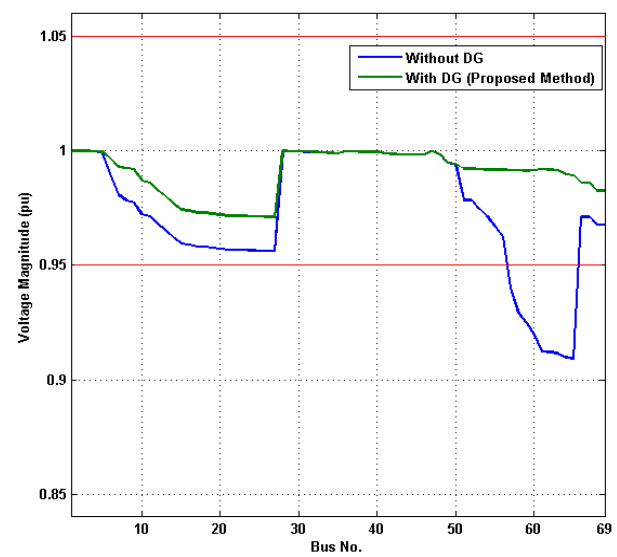

(d) 69-Bus System

Fig. 4. Voltage Profile Improvement due to DG and Capacitor Placement 
From Fig. 4, it can be seen that the overall voltage profile has been significantly improved. Further the voltage levels are in allowable maximum and minimum levels.

\section{CONCLUSION}

This paper has presented a new approach for optimum DG and shunt capacitor placement simultaneously. The proposed method is tested on several radial distribution networks. The result shows that the proposed methods has significantly reduced the power system losses as well as improve the overall loading factor. The system voltage profile has also been significantly improved and all busses found operating in allowable operating limit.

\section{APPENDIX}

Radial distribution test systems 12-bus [11], 30-bus [12], 33-bus [13] and 69-bus [14] data.

TABLE A-I: 12 BUS-SYSTEM DATA

\begin{tabular}{ccccccc}
\hline $\begin{array}{c}\text { Line } \\
\text { No. }\end{array}$ & From & To & $\mathrm{R}(\mathrm{pu})$ & $\mathrm{X}(\mathrm{pu})$ & $\mathrm{P}_{\mathrm{L}}(\mathrm{kW})$ & $\mathrm{Q}_{\mathrm{L}}(\mathrm{kW})$ \\
\hline 1 & 1 & 2 & 0.00009 & 0.000038 & 0.06 & 0.06 \\
2 & 2 & 3 & 0.000098 & 0.000041 & 0.04 & 0.03 \\
3 & 3 & 4 & 0.000173 & 0.000072 & 0.055 & 0.055 \\
4 & 4 & 5 & 0.000263 & 0.00011 & 0.03 & 0.03 \\
5 & 5 & 6 & 0.00009 & 0.000038 & 0.02 & 0.015 \\
6 & 6 & 7 & 0.000083 & 0.000034 & 0.055 & 0.055 \\
7 & 7 & 8 & 0.000364 & 0.0001 & 0.045 & 0.045 \\
8 & 8 & 9 & 0.000466 & 0.000132 & 0.04 & 0.04 \\
9 & 9 & 10 & 0.000239 & 0.000068 & 0.035 & 0.03 \\
10 & 10 & 11 & 0.000125 & 0.000035 & 0.04 & 0.03 \\
11 & 11 & 12 & 0.000102 & 0.000029 & 0.015 & 0.015 \\
\hline \hline
\end{tabular}

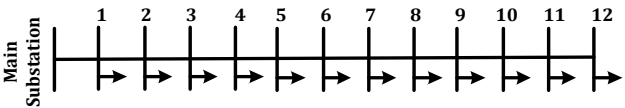

Fig. A-1. 12-Bus radial distribution system

TABLE A-II: 30 BUS-SYSTEM DATA

\begin{tabular}{|c|c|c|c|c|c|c|}
\hline $\begin{array}{l}\text { Line } \\
\text { No. }\end{array}$ & From & To & $\mathrm{R}(\mathrm{pu})$ & $\mathrm{X}(\mathrm{pu})$ & $\mathrm{P}_{\mathrm{L}}(\mathrm{kW})$ & $\mathrm{Q}_{\mathrm{L}}(\mathrm{kW})$ \\
\hline 1 & 1 & 2 & 0.0967 & 0.0397 & 0.4182 & 0.2591 \\
\hline 2 & 2 & 3 & 0.0886 & 0.0364 & 0 & 0 \\
\hline 3 & 3 & 4 & 0.1359 & 0.0377 & 0.4182 & 0.2591 \\
\hline 4 & 4 & 5 & 0.1236 & 0.0343 & 0.4182 & 0.2591 \\
\hline 5 & 5 & 6 & 0.1236 & 0.0343 & 0 & 0 \\
\hline 6 & 6 & 7 & 0.2598 & 0.0446 & 0 & 0 \\
\hline 7 & 7 & 8 & 0.1732 & 0.0298 & 0.4182 & 0.2591 \\
\hline 8 & 8 & 9 & 0.2598 & 0.0446 & 0.4182 & 0.2591 \\
\hline 9 & 9 & 10 & 0.1732 & 0.0298 & 0.4071 & 0.2464 \\
\hline 10 & 10 & 11 & 0.1083 & 0.0186 & 0.4182 & 0.2591 \\
\hline 11 & 11 & 12 & 0.0866 & 0.0149 & 0.2491 & 0.1527 \\
\hline 12 & 3 & 13 & 0.1299 & 0.0223 & 0.1143 & 0.0714 \\
\hline 13 & 13 & 14 & 0.1732 & 0.0298 & 0.1143 & 0.0714 \\
\hline 14 & 14 & 15 & 0.0866 & 0.0149 & 0.1143 & 0.0714 \\
\hline 15 & 15 & 16 & 0.0433 & 0.0074 & 0.0214 & 0.0119 \\
\hline 16 & 6 & 17 & 0.1483 & 0.0412 & 0.4423 & 0.274 \\
\hline 17 & 17 & 18 & 0.1359 & 0.0377 & 0.4423 & 0.274 \\
\hline 18 & 18 & 19 & 0.1718 & 0.0391 & 0.4423 & 0.274 \\
\hline 19 & 19 & 20 & 0.1562 & 0.0355 & 0.4423 & 0.274 \\
\hline 20 & 20 & 21 & 0.1562 & 0.0355 & 0.4423 & 0.274 \\
\hline 21 & 21 & 22 & 0.2165 & 0.0372 & 0.4423 & 0.274 \\
\hline 22 & 22 & 23 & 0.2165 & 0.0372 & 0.4423 & 0.274 \\
\hline 23 & 23 & 24 & 0.2598 & 0.0446 & 0.4423 & 0.274 \\
\hline 24 & 24 & 25 & 0.1732 & 0.0298 & 0.4423 & 0.274 \\
\hline 25 & 25 & 26 & 0.1083 & 0.0186 & 0.4423 & 0.274 \\
\hline 26 & 26 & 27 & 0.0866 & 0.0149 & 0.2635 & 0.1635 \\
\hline 27 & 7 & 28 & 0.1299 & 0.0223 & 0.1667 & 0.1067 \\
\hline 28 & 28 & 29 & 0.1299 & 0.0223 & 0.1667 & 0.1067 \\
\hline 29 & 29 & 30 & 0.1299 & 0.0223 & 0.1667 & 0.1067 \\
\hline
\end{tabular}

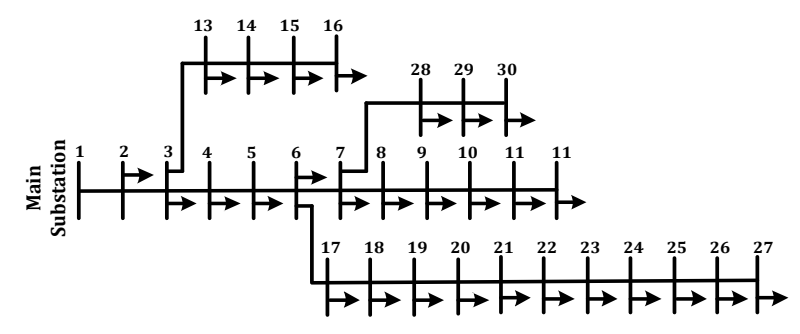

Fig. A-2. 30-Bus radial distribution system

TABLE A-III: 33 BUS-SYSTEM DATA

\begin{tabular}{rrrrrrr}
\hline \hline $\begin{array}{r}\text { Line } \\
\text { No. }\end{array}$ & From & To & \multicolumn{1}{c}{$\mathrm{R}(\mathrm{pu})$} & $\mathrm{X}(\mathrm{pu})$ & $\mathrm{P}_{\mathrm{L}}(\mathrm{kW})$ & $\mathrm{Q}_{\mathrm{L}}(\mathrm{kW})$ \\
\hline 1 & 1 & 2 & 0.0575 & 0.0293 & 0.1 & 0.06 \\
2 & 2 & 3 & 0.3076 & 0.1566 & 0.09 & 0.04 \\
3 & 3 & 4 & 0.2284 & 0.1163 & 0.12 & 0.08 \\
4 & 4 & 5 & 0.2378 & 0.1211 & 0.06 & 0.03 \\
5 & 5 & 6 & 0.511 & 0.4411 & 0.06 & 0.02 \\
6 & 6 & 7 & 0.1168 & 0.3861 & 0.2 & 0.1 \\
7 & 7 & 8 & 1.068 & 0.771 & 0.2 & 0.1 \\
8 & 8 & 9 & 0.6426 & 0.4617 & 0.06 & 0.02 \\
9 & 9 & 10 & 0.6514 & 0.4617 & 0.06 & 0.02 \\
10 & 10 & 11 & 0.1227 & 0.0406 & 0.045 & 0.03 \\
11 & 11 & 12 & 0.2336 & 0.0772 & 0.06 & 0.035 \\
12 & 12 & 13 & 0.9159 & 0.7206 & 0.06 & 0.035 \\
13 & 13 & 14 & 0.3379 & 0.4448 & 0.12 & 0.08 \\
14 & 14 & 15 & 0.3687 & 0.3282 & 0.06 & 0.01 \\
15 & 15 & 16 & 0.4656 & 0.34 & 0.06 & 0.02 \\
16 & 16 & 17 & 0.8042 & 1.0738 & 0.06 & 0.02 \\
17 & 17 & 18 & 0.4567 & 0.3581 & 0.09 & 0.04 \\
18 & 2 & 19 & 0.1023 & 0.0976 & 0.09 & 0.04 \\
19 & 19 & 20 & 0.9385 & 0.8457 & 0.09 & 0.04 \\
20 & 20 & 21 & 0.2555 & 0.2985 & 0.09 & 0.04 \\
21 & 21 & 22 & 0.4423 & 0.5848 & 0.09 & 0.04 \\
22 & 3 & 23 & 0.2815 & 0.1924 & 0.09 & 0.05 \\
23 & 23 & 24 & 0.5603 & 0.4424 & 0.42 & 0.2 \\
24 & 24 & 25 & 0.559 & 0.4374 & 0.42 & 0.2 \\
25 & 6 & 26 & 0.1267 & 0.0645 & 0.06 & 0.025 \\
26 & 26 & 27 & 0.1773 & 0.0903 & 0.06 & 0.025 \\
27 & 27 & 28 & 0.6607 & 0.5826 & 0.06 & 0.02 \\
28 & 28 & 29 & 0.5018 & 0.4371 & 0.12 & 0.07 \\
29 & 29 & 30 & 0.3166 & 0.1613 & 0.2 & 0.6 \\
30 & 30 & 31 & 0.608 & 0.6008 & 0.15 & 0.07 \\
31 & 31 & 32 & 0.1937 & 0.2258 & 0.21 & 0.1 \\
32 & 32 & 33 & 0.2128 & 0.3308 & 0.06 & 0.04 \\
\hline \hline & & & & & &
\end{tabular}

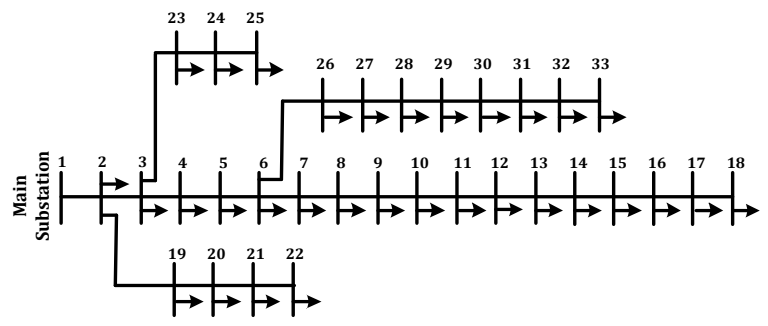

Fig. A-3. 33-Bus radial distribution system

TABLE A-IV: 69 BUS-SYSTEM DATA

\begin{tabular}{ccccccc}
\hline $\begin{array}{c}\text { Line } \\
\text { No. }\end{array}$ & From & To & $\mathrm{R}(\mathrm{pu})$ & $\mathrm{X}(\mathrm{pu})$ & $\mathrm{P}_{\mathrm{L}}(\mathrm{kW})$ & $\mathrm{Q}_{\mathrm{L}}(\mathrm{kW})$ \\
\hline 1 & 1 & 2 & 0.0003 & 0.0007 & 0 & 0 \\
2 & 2 & 3 & 0.0003 & 0.0007 & 0 & 0 \\
3 & 3 & 4 & 0.0009 & 0.0022 & 0 & 0 \\
4 & 4 & 5 & 0.0157 & 0.0183 & 0 & 0 \\
5 & 5 & 6 & 0.2284 & 0.1163 & 0.0026 & 0.0022 \\
6 & 6 & 7 & 0.2378 & 0.1211 & 0.0404 & 0.03 \\
7 & 7 & 8 & 0.0575 & 0.0293 & 0.075 & 0.054 \\
8 & 8 & 9 & 0.0308 & 0.0157 & 0.03 & 0.022 \\
9 & 9 & 10 & 0.511 & 0.1689 & 0.028 & 0.019 \\
10 & 10 & 11 & 0.1168 & 0.0386 & 0.145 & 0.104 \\
11 & 11 & 12 & 0.4438 & 0.1467 & 0.145 & 0.104 \\
12 & 12 & 13 & 0.6426 & 0.2121 & 0.008 & 0.0055 \\
13 & 13 & 14 & 0.6514 & 0.2152 & 0.008 & 0.0055
\end{tabular}




$\begin{array}{ccccccc}14 & 14 & 15 & 0.6601 & 0.2181 & 0 & 0 \\ 15 & 15 & 16 & 0.1227 & 0.0406 & 0.0455 & 0.03 \\ 16 & 16 & 17 & 0.2336 & 0.0772 & 0.06 & 0.035 \\ 17 & 17 & 18 & 0.0029 & 0.001 & 0.06 & 0.035 \\ 18 & 18 & 19 & 0.2044 & 0.0676 & 0 & 0 \\ 19 & 19 & 20 & 0.1314 & 0.0434 & 0.001 & 0.0006 \\ 20 & 20 & 21 & 0.2131 & 0.0704 & 0.114 & 0.081 \\ 21 & 21 & 22 & 0.0087 & 0.0029 & 0.0053 & 0.0035 \\ 22 & 22 & 23 & 0.0993 & 0.0328 & 0 & 0 \\ 23 & 23 & 24 & 0.2161 & 0.0714 & 0.028 & 0.02 \\ 24 & 24 & 25 & 0.4672 & 0.1544 & 0 & 0 \\ 25 & 25 & 26 & 0.1927 & 0.0637 & 0.014 & 0.01 \\ 26 & 26 & 27 & 0.1081 & 0.0357 & 0.014 & 0.01 \\ 27 & 3 & 28 & 0.0027 & 0.0067 & 0.026 & 0.0185 \\ 28 & 28 & 29 & 0.0399 & 0.0976 & 0.026 & 0.0185 \\ 29 & 29 & 30 & 0.2482 & 0.082 & 0 & 0 \\ 30 & 30 & 31 & 0.0438 & 0.0145 & 0 & 0 \\ 31 & 31 & 32 & 0.219 & 0.0724 & 0 & 0 \\ 32 & 32 & 33 & 0.5235 & 0.1757 & 0.014 & 0.01 \\ 33 & 33 & 34 & 1.0656 & 0.3523 & 0.0195 & 0.014 \\ 34 & 34 & 35 & 0.9196 & 0.304 & 0.006 & 0.004 \\ 35 & 3 & 36 & 0.0027 & 0.0067 & 0.026 & 0.0186 \\ 36 & 36 & 37 & 0.0399 & 0.0976 & 0.026 & 0.0186 \\ 37 & 37 & 38 & 0.0657 & 0.0767 & 0 & 0 \\ 38 & 38 & 39 & 0.019 & 0.0221 & 0.024 & 0.017 \\ 39 & 39 & 40 & 0.0011 & 0.0013 & 0.024 & 0.017 \\ 40 & 40 & 41 & 0.4544 & 0.5309 & 0.0012 & 0.001 \\ 41 & 41 & 42 & 0.1934 & 0.226 & 0 & 0 \\ 42 & 42 & 43 & 0.0256 & 0.0298 & 0.006 & 0.0043\end{array}$

\begin{tabular}{ccccccc}
43 & 43 & 44 & 0.0057 & 0.0072 & 0 & 0 \\
44 & 44 & 45 & 0.0679 & 0.0857 & 0.0392 & 0.0263 \\
45 & 45 & 46 & 0.0006 & 0.0007 & 0.0392 & 0.0263 \\
46 & 4 & 47 & 0.0021 & 0.0052 & 0 & 0 \\
47 & 47 & 48 & 0.0531 & 0.13 & 0.079 & 0.0564 \\
48 & 48 & 49 & 0.1808 & 0.4424 & 0.3847 & 0.2745 \\
49 & 49 & 50 & 0.0513 & 0.1255 & 0.3847 & 0.2745 \\
50 & 8 & 51 & 0.0579 & 0.0295 & 0.0405 & 0.0283 \\
51 & 51 & 52 & 0.2071 & 0.0695 & 0.0036 & 0.0027 \\
52 & 9 & 53 & 0.1086 & 0.0553 & 0.0043 & 0.0035 \\
53 & 53 & 54 & 0.1267 & 0.0645 & 0.0264 & 0.019 \\
54 & 54 & 55 & 0.1773 & 0.0903 & 0.024 & 0.0172 \\
55 & 55 & 56 & 0.1755 & 0.0894 & 0 & 0 \\
56 & 56 & 57 & 0.992 & 0.333 & 0 & 0 \\
57 & 57 & 58 & 0.489 & 0.1641 & 0 & 0 \\
58 & 58 & 59 & 0.1898 & 0.0628 & 0.1 & 0.072 \\
59 & 59 & 60 & 0.2409 & 0.0731 & 0 & 0 \\
60 & 60 & 61 & 0.3166 & 0.1613 & 1.244 & 0.888 \\
61 & 61 & 62 & 0.0608 & 0.0309 & 0.032 & 0.023 \\
62 & 62 & 63 & 0.0905 & 0.046 & 0 & 0 \\
63 & 63 & 64 & 0.4433 & 0.2258 & 0.227 & 0.162 \\
64 & 64 & 65 & 0.6495 & 0.3308 & 0.059 & 0.042 \\
65 & 11 & 66 & 0.1255 & 0.0381 & 0.018 & 0.013 \\
66 & 66 & 67 & 0.0029 & 0.0009 & 0.018 & 0.013 \\
67 & 12 & 68 & 0.4613 & 0.1525 & 0.028 & 0.02 \\
68 & 68 & 69 & 0.0029 & 0.001 & 0.028 & 0.02 \\
\hline \hline$* \mathrm{P}_{\mathrm{L}}$ and $\mathrm{Q}_{\mathrm{L}}$ represent receiving end bus power & & \\
\hline
\end{tabular}

$* \mathrm{P}_{\mathrm{L}}$ and $\mathrm{Q}_{\mathrm{L}}$ represent receiving end bus power.

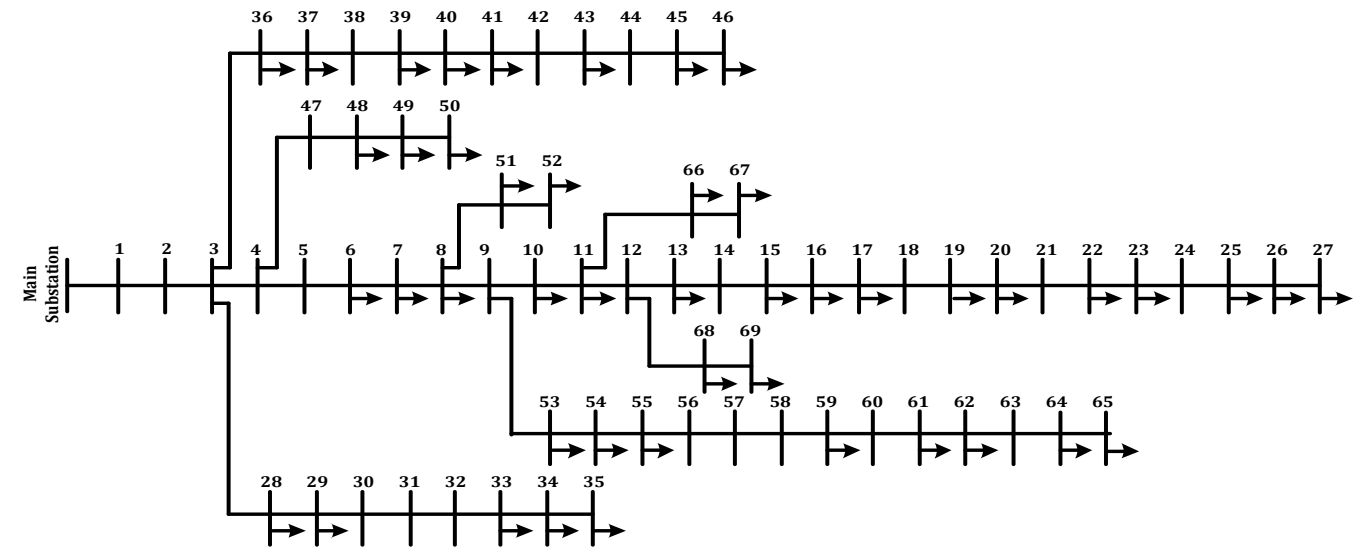

Fig. A-4. 69-Bus radial distribution system

Without DG and shunt capacitor placement, load flow analysis results are as follows:

TABLE A-V: 12 BUS-SYSTEM DATA

\begin{tabular}{cccccc}
\multicolumn{6}{c}{ TABLE A-V: 12 Bus-SYSTEM DATA } \\
\hline \hline Bus & \multirow{2}{*}{$\mathrm{V}(\mathrm{pu})$} & \multirow{2}{*}{ From } & \multirow{2}{*}{ To } & \multicolumn{2}{c}{ Line Losses } \\
\cline { 5 - 6 } No. & & & & $\mathrm{P}$ & $\mathrm{Q}$ \\
\hline 1 & 1 & 1 & 2 & 0.003404 & 0.001437 \\
2 & 0.994331 & 2 & 3 & 0.002751 & 0.001151 \\
3 & 0.989017 & 3 & 4 & 0.003977 & 0.001655 \\
4 & 0.980574 & 4 & 5 & 0.004213 & 0.001762 \\
5 & 0.969829 & 5 & 6 & 0.001144 & 0.000483 \\
6 & 0.966542 & 6 & 7 & 0.000908 & 0.000372 \\
7 & 0.963761 & 7 & 8 & 0.002278 & 0.000626 \\
8 & 0.955326 & 8 & 9 & 0.001572 & 0.000445 \\
9 & 0.947297 & 9 & 10 & 0.000368 & 0.000105 \\
10 & 0.944477 & 10 & 11 & $7.09 \mathrm{E}-05$ & $1.99 \mathrm{E}-05$ \\
11 & 0.943581 & 11 & 12 & $5.16 \mathrm{E}-06$ & $1.47 \mathrm{E}-06$ \\
\hline 12 & 0.943373 & \multicolumn{7}{c}{ Total } & $\mathbf{0 . 0 2 0 7}$ & $\mathbf{0 . 0 0 8 1}$ \\
\hline \hline
\end{tabular}

TABLE A-VI: 30-BUS SYSTEM DATA

\begin{tabular}{cccccc}
\hline \hline Bus & \multirow{2}{*}{ V (pu) } & \multirow{2}{*}{ From } & \multirow{2}{*}{ To } & \multicolumn{2}{c}{ Line Losses } \\
No. & & 1 & 2 & 0.12008 & 0.049299 \\
\hline 1 & 1 & 2 & 3 & 0.100415 & 0.041254 \\
2 & 0.988472 & 2 & 4 & 0.141594 & 0.03928 \\
3 & 0.978381 & 3 & & &
\end{tabular}

\begin{tabular}{ccccc}
0.964491 & 4 & 5 & 0.116232 & 0.032255 \\
0.952497 & 5 & 6 & 0.104182 & 0.028911 \\
0.94115 & 6 & 7 & 0.024389 & 0.004187 \\
0.933652 & 7 & 8 & 0.010204 & 0.001756 \\
0.929688 & 8 & 9 & 0.00936 & 0.001607 \\
0.92504 & 9 & 10 & 0.003233 & 0.000556 \\
0.922809 & 10 & 11 & 0.000784 & 0.000135 \\
0.921941 & 11 & 12 & $8.70 \mathrm{E}-05$ & $1.50 \mathrm{E}-05$ \\
0.921683 & 3 & 13 & 0.00025 & $4.29 \mathrm{E}-05$ \\
0.977846 & 13 & 14 & 0.000157 & $2.70 \mathrm{E}-05$ \\
0.977355 & 14 & 15 & $2.30 \mathrm{E}-05$ & $3.96 \mathrm{E}-06$ \\
0.977222 & 15 & 16 & $2.72 \mathrm{E}-07$ & $4.65 \mathrm{E}-08$ \\
0.977212 & 6 & 17 & 0.055492 & 0.015416 \\
0.932078 & 17 & 18 & 0.041995 & 0.01165 \\
0.924528 & 18 & 19 & 0.042884 & 0.00976 \\
0.916174 & 19 & 20 & 0.030631 & 0.006961 \\
0.909447 & 20 & 21 & 0.023228 & 0.005279 \\
0.903591 & 21 & 22 & 0.023298 & 0.004003 \\
0.896896 & 22 & 23 & 0.015787 & 0.002713 \\
0.891389 & 23 & 24 & 0.01164 & 0.001998 \\
0.886213 & 24 & 25 & 0.004052 & 0.000697 \\
0.88372 & 25 & 26 & 0.000959 & 0.000165 \\
0.882761 & 26 & 27 & 0.000107 & $1.84 \mathrm{E}-05$ \\
0.882475 & 7 & 28 & 0.000527 & $9.04 \mathrm{E}-05$ \\
0.932878 & 28 & 29 & 0.000234 & $4.02 \mathrm{E}-05$ \\
0.932363 & 29 & 30 & $5.86 \mathrm{E}-05$ & $1.01 \mathrm{E}-05$ \\
\cline { 2 - 4 } 0.932105 & Total & $\mathbf{0 . 8 8 1 9}$ & $\mathbf{0 . 2 5 8 1}$ \\
\hline \multicolumn{5}{c}{}
\end{tabular}


TABLE A-VI: 33-BUS SYSTEM DATA

\begin{tabular}{|c|c|c|c|c|c|}
\hline \multirow{2}{*}{$\begin{array}{l}\text { Bus } \\
\text { No. }\end{array}$} & \multirow{2}{*}{$\mathrm{V}(\mathrm{pu})$} & \multirow[t]{2}{*}{ From } & \multirow[t]{2}{*}{ To } & \multicolumn{2}{|c|}{ Line Losses } \\
\hline & & & & $\mathrm{P}$ & Q \\
\hline 1 & 1 & 1 & 2 & 0.012294 & 0.006265 \\
\hline 2 & 0.997027 & 2 & 3 & 0.052073 & 0.026511 \\
\hline 3 & 0.982896 & 3 & 4 & 0.020055 & 0.010212 \\
\hline 4 & 0.975386 & 4 & 5 & 0.01885 & 0.009599 \\
\hline 5 & 0.96796 & 5 & 6 & 0.038562 & 0.033287 \\
\hline 6 & 0.949483 & 6 & 7 & 0.001946 & 0.006433 \\
\hline 7 & 0.945958 & 7 & 8 & 0.011875 & 0.008572 \\
\hline 8 & 0.932299 & 8 & 9 & 0.004265 & 0.003064 \\
\hline 9 & 0.925967 & 9 & 10 & 0.003633 & 0.002575 \\
\hline 10 & 0.920093 & 10 & 11 & 0.000565 & 0.000187 \\
\hline 11 & 0.919224 & 11 & 12 & 0.000899 & 0.000297 \\
\hline 12 & 0.917709 & 12 & 13 & 0.002721 & 0.002141 \\
\hline 13 & 0.911534 & 13 & 14 & 0.000744 & 0.000979 \\
\hline 14 & 0.909244 & 14 & 15 & 0.000364 & 0.000324 \\
\hline 15 & 0.907817 & 15 & 16 & 0.000287 & 0.00021 \\
\hline 16 & 0.906436 & 16 & 17 & 0.000257 & 0.000343 \\
\hline 17 & $9.04 \mathrm{E}-01$ & 17 & 18 & $5.42 \mathrm{E}-05$ & $4.25 \mathrm{E}-05$ \\
\hline 18 & 0.903774 & 2 & 19 & 0.000161 & 0.000154 \\
\hline 19 & 0.996499 & 19 & 20 & 0.000832 & 0.00075 \\
\hline 20 & 0.992921 & 20 & 21 & 0.000101 & 0.000118 \\
\hline 21 & $9.92 \mathrm{E}-01$ & 21 & 22 & $4.36 \mathrm{E}-05$ & 5.77E-05 \\
\hline 22 & 0.991579 & 3 & 23 & 0.003182 & 0.002175 \\
\hline 23 & 0.979311 & 23 & 24 & 0.005144 & 0.004062 \\
\hline 24 & 0.972639 & 24 & 25 & 0.001287 & 0.001007 \\
\hline 25 & 0.969314 & 6 & 26 & 0.002603 & 0.001325 \\
\hline 26 & 0.947553 & 26 & 27 & 0.00333 & 0.001696 \\
\hline 27 & 0.944989 & 27 & 28 & 0.011304 & 0.009968 \\
\hline 28 & 0.933547 & 28 & 29 & 0.007836 & 0.006826 \\
\hline 29 & 0.925328 & 29 & 30 & 0.003896 & 0.001985 \\
\hline 30 & 0.92177 & 30 & 31 & 0.001594 & 0.001575 \\
\hline 31 & 0.917608 & 31 & 32 & 0.000213 & 0.000249 \\
\hline 32 & $9.17 \mathrm{E}-01$ & 32 & 33 & $1.32 \mathrm{E}-05$ & $2.05 \mathrm{E}-05$ \\
\hline 33 & 0.916409 & & & 0.2110 & 0.1430 \\
\hline
\end{tabular}

TABLE A-VI: 69-BUS SYSTEM DATA

\begin{tabular}{|c|c|c|c|c|c|}
\hline \multirow{2}{*}{$\begin{array}{l}\text { Bus } \\
\text { No. }\end{array}$} & \multirow{2}{*}{$\mathrm{V}(\mathrm{pu})$} & \multirow{2}{*}{ From } & \multirow{2}{*}{ To } & \multicolumn{2}{|c|}{ Line Losses } \\
\hline & & & & $\mathrm{P}$ & Q \\
\hline 1 & 1 & 1 & 2 & $7.21 \mathrm{E}-05$ & 0.000168 \\
\hline 2 & 0.999968 & 2 & 3 & $7.21 \mathrm{E}-05$ & 0.000168 \\
\hline 3 & 0.999937 & 3 & 4 & 0.000187 & 0.000458 \\
\hline 4 & 0.999846 & 4 & 5 & 0.001942 & 0.002263 \\
\hline 5 & 0.999026 & 5 & 6 & 0.028249 & 0.014384 \\
\hline 6 & 0.99009 & 6 & 7 & 0.029354 & 0.014949 \\
\hline 7 & 0.980796 & 7 & 8 & 0.006892 & 0.003512 \\
\hline 8 & 0.978581 & 8 & 9 & 0.00338 & 0.001723 \\
\hline 9 & 0.977446 & 9 & 10 & 0.004778 & 0.001579 \\
\hline 10 & 0.972445 & 10 & 11 & 0.001015 & 0.000335 \\
\hline 11 & 0.971344 & 11 & 12 & 0.002192 & 0.000725 \\
\hline 12 & 0.968183 & 12 & 13 & 0.001287 & 0.000425 \\
\hline 13 & 0.965257 & 13 & 14 & 0.001247 & 0.000412 \\
\hline 14 & 0.962358 & 14 & 15 & 0.001206 & 0.000398 \\
\hline 15 & 0.959488 & 15 & 16 & 0.000224 & $7.42 \mathrm{E}-05$ \\
\hline 16 & 0.958954 & 16 & 17 & 0.000321 & 0.000106 \\
\hline 17 & 0.958074 & 17 & 18 & $2.58 \mathrm{E}-06$ & 8.89E-07 \\
\hline 18 & 0.958065 & 18 & 19 & 0.000104 & $3.45 \mathrm{E}-05$ \\
\hline 19 & 0.9576 & 19 & 20 & $6.71 \mathrm{E}-05$ & $2.22 \mathrm{E}-05$ \\
\hline 20 & 0.957301 & 20 & 21 & 0.000108 & $3.56 \mathrm{E}-05$ \\
\hline 21 & 0.956819 & 21 & 22 & $5.37 \mathrm{E}-07$ & $1.79 \mathrm{E}-07$ \\
\hline 22 & 0.956812 & 22 & 23 & $5.14 \mathrm{E}-06$ & $1.70 \mathrm{E}-06$ \\
\hline 23 & 0.95674 & 23 & 24 & $1.12 \mathrm{E}-05$ & $3.70 \mathrm{E}-06$ \\
\hline 24 & 0.956584 & 24 & 25 & $6.05 \mathrm{E}-06$ & $2.00 \mathrm{E}-06$ \\
\hline 25 & 0.956415 & 25 & 26 & $2.49 \mathrm{E}-06$ & $8.25 \mathrm{E}-07$ \\
\hline 26 & 0.956345 & 26 & 27 & $3.50 \mathrm{E}-07$ & $1.16 \mathrm{E}-07$ \\
\hline 27 & 0.956325 & 3 & 28 & $3.40 \mathrm{E}-07$ & $8.45 \mathrm{E}-07$ \\
\hline 28 & 0.99993 & 28 & 29 & $2.58 \mathrm{E}-06$ & $6.30 \mathrm{E}-06$ \\
\hline 29 & 0.999858 & 29 & 30 & $5.83 \mathrm{E}-06$ & $1.93 \mathrm{E}-06$ \\
\hline 30 & 0.999737 & 30 & 31 & $1.03 \mathrm{E}-06$ & $3.41 \mathrm{E}-07$ \\
\hline 31 & 0.999716 & 31 & 32 & $5.14 \mathrm{E}-06$ & $1.70 \mathrm{E}-06$ \\
\hline 32 & 0.999609 & 32 & 33 & $1.23 \mathrm{E}-05$ & 4.13E-06 \\
\hline 33 & 0.999353 & 33 & 34 & $1.04 \mathrm{E}-05$ & $3.44 \mathrm{E}-06$ \\
\hline 34 & 0.999017 & 34 & 35 & 4.79E-07 & $1.58 \mathrm{E}-07$ \\
\hline 35 & 0.99895 & 3 & 36 & $1.38 \mathrm{E}-06$ & $3.43 \mathrm{E}-06$ \\
\hline 36 & 0.999923 & 36 & 37 & $1.51 \mathrm{E}-05$ & $3.69 \mathrm{E}-05$ \\
\hline 37 & 0.999751 & 37 & 38 & $1.73 \mathrm{E}-05$ & $2.02 \mathrm{E}-05$ \\
\hline
\end{tabular}

\begin{tabular}{|c|c|c|c|c|c|}
\hline \multirow{2}{*}{$\begin{array}{l}\text { Bus } \\
\text { No. }\end{array}$} & \multirow{2}{*}{$\mathrm{V}(\mathrm{pu})$} & \multirow{2}{*}{ From } & \multirow{2}{*}{ To } & \multicolumn{2}{|c|}{ Line Losses } \\
\hline & & & & $\mathrm{P}$ & Q \\
\hline 38 & 0.999593 & 38 & 39 & $5.01 \mathrm{E}-06$ & $5.82 \mathrm{E}-06$ \\
\hline 39 & 0.999547 & 39 & 40 & $1.94 \mathrm{E}-07$ & $2.30 \mathrm{E}-07$ \\
\hline 40 & 0.999545 & 40 & 41 & $4.87 \mathrm{E}-05$ & $5.69 \mathrm{E}-05$ \\
\hline 41 & 0.998847 & 41 & 42 & $2.01 \mathrm{E}-05$ & 2.35E-05 \\
\hline 42 & 0.998555 & 42 & 43 & $2.66 \mathrm{E}-06$ & $3.10 \mathrm{E}-06$ \\
\hline 43 & 0.998516 & 43 & 44 & $5.10 \mathrm{E}-07$ & $6.44 \mathrm{E}-07$ \\
\hline 44 & 0.998508 & 44 & 45 & $6.07 \mathrm{E}-06$ & $7.66 \mathrm{E}-06$ \\
\hline 45 & 0.99841 & 45 & 46 & $1.34 \mathrm{E}-08$ & $1.56 \mathrm{E}-08$ \\
\hline 46 & 0.998409 & 4 & 47 & 2.31E-05 & $5.71 \mathrm{E}-05$ \\
\hline 47 & 0.999796 & 47 & 48 & 0.000583 & 0.001427 \\
\hline 48 & 0.99855 & 48 & 49 & 0.001633 & 0.003997 \\
\hline 49 & 0.994705 & 49 & 50 & 0.000116 & 0.000284 \\
\hline 50 & 0.99416 & 8 & 51 & $1.76 \mathrm{E}-06$ & $8.95 \mathrm{E}-07$ \\
\hline 51 & 0.978545 & 51 & 52 & 4.38E-08 & $1.47 \mathrm{E}-08$ \\
\hline 52 & 0.978536 & 9 & 53 & 0.005783 & 0.002945 \\
\hline 53 & 0.974658 & 53 & 54 & 0.006714 & 0.003418 \\
\hline 54 & 0.971415 & 54 & 55 & 0.009124 & 0.004647 \\
\hline 55 & 0.966941 & 55 & 56 & 0.008789 & 0.004477 \\
\hline 56 & 0.962573 & 56 & 57 & 0.049682 & 0.016677 \\
\hline 57 & 0.9401 & 57 & 58 & 0.02449 & 0.008219 \\
\hline 58 & 0.92904 & 58 & 59 & 0.009506 & 0.003145 \\
\hline 59 & 0.924762 & 59 & 60 & 0.010671 & 0.003238 \\
\hline 60 & 0.919737 & 60 & 61 & 0.014024 & 0.007145 \\
\hline 61 & 0.912341 & 61 & 62 & 0.000112 & $5.70 \mathrm{E}-05$ \\
\hline 62 & 0.912051 & 62 & 63 & 0.000135 & $6.86 \mathrm{E}-05$ \\
\hline 63 & 0.911664 & 63 & 64 & 0.000661 & 0.000337 \\
\hline 64 & 0.909763 & 64 & 65 & 4.12E-05 & $2.10 \mathrm{E}-05$ \\
\hline 65 & 0.909189 & 11 & 66 & $2.62 \mathrm{E}-06$ & $7.96 \mathrm{E}-07$ \\
\hline 66 & 0.971287 & 66 & 67 & $1.52 \mathrm{E}-08$ & 4.70E-09 \\
\hline 67 & 0.971286 & 12 & 68 & 2.33E-05 & 7.71E-06 \\
\hline 68 & 0.967853 & 68 & 69 & $3.67 \mathrm{E}-08$ & $1.26 \mathrm{E}-08$ \\
\hline 69 & 0.967852 & & & 0.2250 & 0.1021 \\
\hline
\end{tabular}

\section{ACKNOWLEDGMENT}

The first author gratefully acknowledges the support given by NED University of Engineering and Technology Pakistan and the Bright Spark Programme of University of Malaya.

\section{REFERENCES}

[1] T. Ackermann, G. Andersson, and L. Söder, "Distributed generation: a definition," Electric Power Systems Research, vol. 57, pp. 195-204, 2001.

[2] M. M. Aman, G. B. Jasmon, H. Mokhlis, and A. H. A. Bakar, "Optimal placement and sizing of a DG based on a new power stability index and line losses," International Journal of Electrical Power \&amp; Energy Systems, vol. 43, pp. 1296-1304, 2012.

[3] T. Lund, "Analysis of distribution systems with a high penetration of distributed generation," Centre for Electric Technology (CET), Technical University of Denmark, 2007.

[4] H. M. Khodr, F. G. Olsina, P. M. D. O.-D. Jesus, and J. M. Yusta, "Maximum savings approach for location and sizing of capacitors in distribution systems," Electric Power Systems Research, vol. 78, pp. 1192-1203, 2008.

[5] N. Acharya, P. Mahat, and N. Mithulananthan, "An analytical approach for DG allocation in primary distribution network," International Journal of Electrical Power \&amp; Energy Systems, vol. 28, pp. 669-678, 2006.

[6] N. Hadjsaid and D. F. J. F. Canard, "Dispersed generation impact on distribution networks," IEEE Comput Appl Pow, 1999, vol. 12, pp. 22-28.," 1999.

[7] T. Griffin, K. Tomosovic, D. Secrest, and A. Law, "Placement of dispersed generations systems for reduced losses," in Proc. 33rd International conference on sciences, Hawaii, 2000.

[8] G. Andersson, P. Donalek, R. Farmer, N. Hatziargyriou, I. Kamwa, P. Kundur, N. Martins, J. Paserba, P. Pourbeik, J. Sanchez-Gasca, R. Schulz, A. Stankovic, C. Taylor, and V. Vittal, "Causes of the 2003 major grid blackouts in North America and Europe, and recommended means to improve system dynamic performance," IEEE Transactions on Power Systems, vol. 20, pp. 1922-1928, 2005.

[9] L. Pereira, "Cascade to black [system blackouts]," Power and Energy Magazine, IEEE, vol. 2, pp. 54-57, 2004. 
[10] J. Kennedy and R. Eberhart, "Particle swarm optimization," in Proc. Neural Networks, IEEE International Conference on, 1995, vol. 4, pp. 1942-1948.

[11] D. Das, H. S. Nagi, and D. P. Kothari, "Novel method for solving radial distribution networks," IEE Proceedings Generation, Transmission and Distribution, vol. 141, pp. 291-298, 1994.

[12] U. Eminoglu and M. H. Hocaoglu, "A new power flow method for radial distribution systems including voltage dependent load models," Electric Power Systems Research, vol. 76, pp. 106-114, 2005.

[13] S. Chandramohan, N. Atturulu, R. P. K. Devi, and B. Venkatesh, "Operating cost minimization of a radial distribution system in a deregulated electricity market through reconfiguration using NSGA method," International journal of electrical power \& energy systems, vol. 32, pp. 126-132, 2010.

[14] M. Baran and F. F. Wu, "Optimal sizing of capacitors placed on a radial distribution system," IEEE Transactions on Power Delivery., vol. 4, pp. 735-743, 1989.

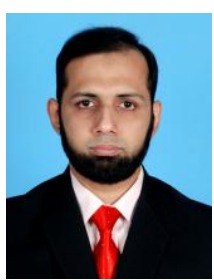

Muhammad Mohsin Aman received his B. Engg. (Bachelors) in Electrical Engineering in 2007 and M. Engg. (Masters) in 2010 from NED University, Karachi, Pakistan. Currently he is pursuing Ph.D. from University of Malaya, Malaysia under the Bright Spark Scholarship Programme. His field of interest is Power System Analysis, Voltage Stability \& Power System Protection. He is a member of IEEE.

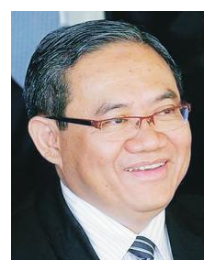

Ghauth Bin Jasmon is the current Vice Chancellor of University of Malaya, Malaysia since November 2008. Prior to this, he was the Multimedia University President for 11 years. Ghauth graduated with a first class honors degree in Electrical Engineering in 1979 and the Ph.D. degree in Power Systems Engineering in 1982 from the University of London. Based on his research, publication and consultancies, Ghauth Jasmon was promoted to Associate Professor in 1989 and to Full Professor in 1992. As an academician, Ghauth Jasmon has contributed extensively in research and in the Engineering Profession. His research areas include Power Systems Analysis, Network Analysis, Voltage
Stability, System Security and Neural Networks. His works have been published in several International journals, conference proceedings and others. He is currently a Senior Member of the IEEE, Fellow of the IET, Fellow of the Institution of Engineers Australia and an Eisenhower Fellow.

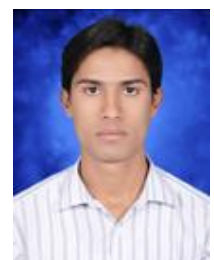

Solangi Khalid Hussain received his B. Engg. (Bachelors) in Mechanical Engineering in 2006 from Quaid-e-Awam University of engineering science and technology (QUEST), Nawabshah, Pakistan. He joined QUEST University in 2008 and still affiliated with the same University. Currently he is pursuing Ph.D. from University of Malaya, Malaysia under QUEST FDP Scholarship Programe. His field of interest is Renewable Energy, Solar energy policies and analysis.

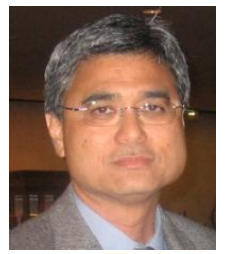

Ab Halim Abu Bakar received his B.Sc. in Electrical Engineering in 1976 from Southampton University UK and $\mathrm{M}$. Eng and $\mathrm{PhD}$ from University Technology Malaysia in 1996 and 2003. $\mathrm{He}$ has 30 years of utility experience in Malaysia before joining academia. Currently he is a consultant in the Department of Electrical Engineering, University of Malaya, Malaysia.

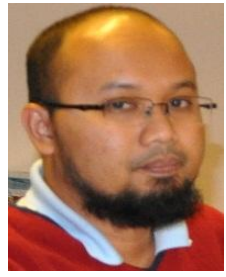

Hazlie Mokhlis received his B. Eng in Electrical Engineering in 1999 and M. Engg Sc in 2002 from University of Malaya, Malaysia. He obtained PhD degree from the University of Manchester, UK in 2009. Currently he is a Lecturer in the Department of Electrical Engineering, University of Malaya. His main research interest is in distribution automation area and power system protection. He is a member of IEEE. 